\title{
High incidence of virulence determinants, aminoglycoside and vancomycin resistance in enterococci isolated from hospitalized patients in Northwest Iran
}

\author{
Fakhri Haghi ${ }^{1}$, Vahid Lohrasbi ${ }^{2,3}$ and Habib Zeighami ${ }^{1,2^{*}}$ (D)
}

\begin{abstract}
Background: Multidrug resistant (MDR) enterococci are important nosocomial pathogens causing serious problem in hospitalized patients. The aim of present study was to investigate the frequency of high-level aminoglycosideresistant and vancomycin-resistant enterococci (VRE) and virulence encoding genes in enterococci isolated from hospitalized patients.
\end{abstract}

Methods: A total of 100 enterococci isolated from urine samples of hospitalized patients with symptomatic urinary tract infections were investigated for antimicrobial susceptibility, the frequency of aminoglycoside and vancomycin

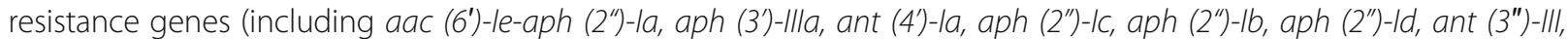
ant ( $\left(6^{\prime}\right)$-la, vanA, vanB and vanC) and virulence encoding genes (including gelE, PAl, esp, ace, cyl, hyl and sprE).

Results: Enterococcus faecalis species was identified as predominant enterococci (69\%), followed by "other" Enterococcus species (21\%) and E. faecium (10\%). Ninety three percent of isolates were resistant to one or more antimicrobial agents, with the most frequent resistance found against tetracycline (86\%), ciprofloxacin (73\%) and quinupristin-dalfopristin (53\%). Gentamicin and streptomycin resistance were detected in 50 and 34\% of isolates, respectively. The most prevalent aminoglycoside resistance genes were ant (3")-III (78\%) and aph (3')-IIIa (67\%). Vancomycin resistance was detected in $21 \%$ of isolates. All E. faecium isolates carried vanA gene, whereas, the vanB gene was not detected in Enterococcus species. The most frequent virulence gene was ace (88.6\%), followed by esp (67.1\%), PAl (45.5\%) and sprE (41.7\%).

Conclusion: Our study revealed the high frequency of gentamycin resistance and VRE in E. faecium isolates, with a high prevalence and heterogeneity of virulence and resistance genes. Due to high frequency of MDR enterococci, it seems that the appropriate surveillance and control measures are essential to prevent the emergence and transmission of these isolates in hospitals.

Keywords: Aminoglycoside resistance, Enterococci, Vancomycin resistance, Virulence factors

\footnotetext{
* Correspondence: zeighami@zums.ac.ir

'Department of Microbiology, School of Medicine, Zanjan University of Medical Sciences, Zanjan, Iran

2Zanjan Pharmaceutical Biotechnology Research Center, Zanjan University of

Medical Sciences, Zanjan, Iran

Full list of author information is available at the end of the article
}

(c) The Author(s). 2019 Open Access This article is distributed under the terms of the Creative Commons Attribution 4.0 International License (http://creativecommons.org/licenses/by/4.0/), which permits unrestricted use, distribution, and reproduction in any medium, provided you give appropriate credit to the original author(s) and the source, provide a link to the Creative Commons license, and indicate if changes were made. The Creative Commons Public Domain Dedication waiver (http://creativecommons.org/publicdomain/zero/1.0/) applies to the data made available in this article, unless otherwise stated. 


\section{Background}

Enterococci are the second most common causative agent of urinary tract infections (UTIs) in hospitalized patients $[1,2]$. Antimicrobial resistance and survival ability in various hospital environments have made them as serious problem in nosocomial infections due to the limited therapeutic options [3, 4]. The inherent antibiotic resistance and dissemination of resistance genes through conjugative transposons and plasmids play an important role in development of multidrug resistant (MDR) enterococci [5].

Aminoglycosides alone are considered inactive in the treatment of enterococcal infections and are usually combined with inhibitors of cell wall synthesis such as vancomycin or ampicillin [6]. High-level aminoglycoside-resistant (HLAR) and vancomycin-resistant enterococci (VRE) have created serious problems for antibiotic therapy [6]. Vancomycin-resistant enterococci are more common in North America, Europe, and Asia. Eight genotypes (vanA, vanB, vanC, vanD, vanE, vanG, vanM and vanL) have been described, of which, $v a n A$ (Tn1546) genotype with acquired inducible resistance to vancomycin and teicoplanin and vanB (Tn1549/Tn5382) genotype with variable resistance to vancomycin and susceptibility to teicoplanin are the most common [7].

High level aminoglycoside resistance is due to acquisition of genes encoding the aminoglycoside modifying enzymes (AMEs) such as aminoglycoside phosphoryl transferase (APH), aminoglycoside acetyl transferase (AAC) and aminoglycoside nucleotidyl transferase (ANT) [8]. The high level gentamicin resistance (HLGR, MIC $\geq 500 \mu \mathrm{g} / \mathrm{ml}$ ) is commonly due to aac (6')-Ie-aph (2")-Ia, which is located on the Tn5281 transposon and encodes a bifunctional enzyme, AAC (6')-APH (2") [8]. Recently, aminoglycoside modifying genes aph (2")-Ib, aph (2")-Ic, and aph (2")-Id were detected among enterococci [9]. These genes are associated with high levels gentamicin resistance. Moreover, high-level streptomycin resistance (HLSR, MIC $\geq 2000 \mu \mathrm{g} / \mathrm{ml}$ ) is mediated by aph (3')-IIIa and ant (6')-Ia genes [9].

Enterococci possess virulence genes including ace, PAI, asa1, sprE, cylA, efaA, esp, gelE and hyl encoding collagenbinding protein, pathogenicity islands, aggregation substance, serine protease, cytolysin, endocarditis antigen, enterococcal surface protein, gelatinase and hyaluronidase, respectively [1]. The gelatinase is an extracellular metalloprotease that hydrolyzes collagen, gelatin, and small peptides [10]. The enterococcal cytolysin is a member of bacteriocin family which lyses bacterial and eukaryotic cells in response to quorum sensing signals [11]. The enterococcal surface protein seems to contribute in the colonization and persistence of enterococci in ascending infections of the urinary tract and biofilm formation. Hyaluronidase is an important factor in nasopharyngeal colonization and pneumonia [10]. Recent studies showed the association between the presence of virulence factors and promoting emergence of enterococcal infections in nosocomial settings [5]. However, our knowledge about the possible relationship between the presence of virulence factors and their role in the emergence and development of resistance among enterococci is still limited [2]. Previous studies revealed that antimicrobial resistance and virulence are two different aspects of bacterial cell fitness and increased antimicrobial resistance might not always be associated with increased virulence [12].

Regarding the emergence of MDR enterococci have become a serious problem in hospitalized patients, the present study aimed to investigate the frequency of HLAR and VRE strains, antibiotic susceptibility, the frequency of AME and Van genes (including aac (6')-Ieaph (2“)-Ia, aph (3')-IIIa, ant (4')-Ia, aph (2”)-Ic, aph (2“)-Ib, aph (2”)-Id, ant (3")-III, ant (6')-Ia, vanA, vanB and $v a n C$ ) and virulence encoding genes (including gelE, PAI, esp, ace, cyl, hyl and sprE) in enterococci isolated from urine samples.

\section{Methods \\ Bacterial isolation and identification}

Between March 2016 and February 2017, 1 hundred enterococci were isolated from urine samples of hospitalized patients with symptomatic urinary tract infections (UTIs) at least $48 \mathrm{~h}$ after hospital admission from three major hospitals in Zanjan, Iran. Informed consent and ethical approval was obtained from management of the hospitals prior to the study. The symptomatic UTI criteria consisted of dysuria, suprapubic pain or tenderness, urgency and frequency of micturition. The exclusion criteria for patients were fever, nausea, vomiting and mixed infection. Catheter urine samples were also excluded from our study. Laboratory confirmed UTI was defined as pyuria (>10 WBC/ $/ \mathrm{mm}^{3}$ per high-power field) plus bacteriuria $\left(\geq 10^{5} \mathrm{cfu} / \mathrm{mL}\right)$. Urine samples were cultured on blood agar (Merck, Germany) and incubated under aerobic conditions at $37^{\circ} \mathrm{C}$ for $24 \mathrm{~h}$. Identification of isolates to the genus level was performed using Gram staining and biochemical tests. Species-level identification was performed by PCR targeting the $d d l$ genes encoding D-alanine-D-alanine ligases specific for E. faecalis $\left(d d l_{E \text {.- }}\right.$ faecalis) and $E$. faecium ( $d d l_{\text {E.faecium }}$ ) (The primers are shown in Table 1). Verified enterococci were preserved at $-70{ }^{\circ} \mathrm{C}$ for further analysis. All Microbiological and molecular tests were performed in department of Microbiology, Zanjan University of Medical Sciences, Zanjan, Iran.

\section{Antimicrobial susceptibility testing}

Susceptibility testing to vancomycin $(30 \mu \mathrm{g})$, ampicillin $(10 \mu \mathrm{g})$, tetracycline $(30 \mu \mathrm{g})$, linezolid $(30 \mu \mathrm{g})$, gentamicin $(120 \mu \mathrm{g})$, chloramphenicol $(30 \mu \mathrm{g})$, fosfomycin $(200 \mu \mathrm{g})$, quinupristin-dalfopristin $(15 \mu \mathrm{g})$, streptomycin $(300 \mu \mathrm{g})$ 
Table 1 Primers sequence and annealing tempratures used in this study

\begin{tabular}{|c|c|c|c|c|}
\hline Target & Primer sequence $\left(5^{\prime} \rightarrow 3^{\prime}\right)$ & Amplicon size (bp) & Annealing tempreture & Ref. \\
\hline \multirow[t]{2}{*}{$d d l_{\text {faecalis }}$} & ATCAAGTACAGTTAGTCTTTATTAG & 941 & $55^{\circ} \mathrm{C}$ & {$[13]$} \\
\hline & ACGATTCAAAGCTAACTGAATCAGT & & & \\
\hline \multirow[t]{2}{*}{$d d l_{\text {faecium }}$} & TTGAGGCAGACCAGATTGACG & 658 & $55^{\circ} \mathrm{C}$ & [13] \\
\hline & TATGACAGCGACTCCGATTCC & & & \\
\hline \multirow[t]{2}{*}{$\operatorname{van} A$} & CATGAATAGAATAAAAGTTGCAATA & $1030 \mathrm{bp}$ & $54^{\circ} \mathrm{C}$ & [14] \\
\hline & CCCCTITAACGCTAATACGATCAA & & & \\
\hline \multirow[t]{2}{*}{$\operatorname{van} B$} & GTGACAAACCGGAGGCGAGGA & $433 \mathrm{bp}$ & $54^{\circ} \mathrm{C}$ & [14] \\
\hline & CCGCCATCCTCCTGCAAAAAA & & & \\
\hline \multirow[t]{2}{*}{$\operatorname{van} C$} & GAAAGACAACAGGAAGACCGC & 796 bp & $54^{\circ} \mathrm{C}$ & [15] \\
\hline & ATCGCATCACAAGCACCAATC & & & \\
\hline \multirow[t]{2}{*}{$\operatorname{aac}\left(6^{\prime}\right)-l e-a p h\left(2^{\prime \prime}\right)-l a$} & CAGGAATTTATCGAAAATGGTAGAAAAG & $369 \mathrm{bp}$ & $55^{\circ} \mathrm{C}$ & [16] \\
\hline & CACAATCGACTAAAGAGTACCAATC & & & \\
\hline \multirow[t]{2}{*}{$a p h\left(3^{\prime}\right)-111 a$} & GGCTAAAATGAGAATATCACCGG & $523 \mathrm{bp}$ & $55^{\circ} \mathrm{C}$ & [16] \\
\hline & CTITAAAAAATCATACAGCTCGCG & & & \\
\hline \multirow[t]{2}{*}{$\operatorname{ant}\left(4^{\prime}\right)-1 a$} & CAAACTGCTAAATCGGTAGAAGCC & 294 bp & $55^{\circ} \mathrm{C}$ & [16] \\
\hline & GGAAAGTTGACCAGACATTACGAACT & & & \\
\hline \multirow[t]{2}{*}{$\operatorname{aph}\left(2^{\prime \prime}\right)-I c$} & CCACAATGATAATGACTCAGTTCCC & $444 \mathrm{bp}$ & $55^{\circ} \mathrm{C}$ & [16] \\
\hline & CCACAGCTTCCGATAGCAAGAG & & & \\
\hline \multirow[t]{2}{*}{$\operatorname{aph}\left(2^{\prime \prime}\right)-1 b$} & CTTGGACGCTGAGATATATGAGCAC & $867 \mathrm{bp}$ & $55^{\circ} \mathrm{C}$ & [16] \\
\hline & GTTTGTAGCAATTCAGAAACACCCTT & & & \\
\hline \multirow[t]{2}{*}{$\operatorname{aph}\left(2^{\prime \prime}\right)-I d$} & GTGGTTITTACAGGAATGCCATC & $641 \mathrm{bp}$ & $55^{\circ} \mathrm{C}$ & [16] \\
\hline & СССТСТTCATACCAATCCATATAACC & & & \\
\hline \multirow[t]{2}{*}{$\operatorname{ant}\left(3^{\prime \prime}\right)-111$} & TGATTTGCTGGTTACGGTGAC & $284 \mathrm{bp}$ & $55^{\circ} \mathrm{C}$ & {$[17]$} \\
\hline & CGCTATGTTCTCTTGCTITTG & & & \\
\hline \multirow[t]{2}{*}{$\operatorname{ant}\left(6^{\prime}\right)-1 a$} & ACTGGCTTAATCAATTTGGG & $596 \mathrm{bp}$ & $55^{\circ} \mathrm{C}$ & {$[17]$} \\
\hline & GCCTTTCCGCCACCTCACCG & & & \\
\hline \multirow[t]{2}{*}{ PAl } & GACGCTCCCTTCTITTGAC & $387 \mathrm{bp}$ & $54^{\circ} \mathrm{C}$ & [18] \\
\hline & CCAGAGAAATTACTACCAT & & & \\
\hline \multirow[t]{2}{*}{ spre } & GGTAAACCAACCAAGTGAATC & $300 \mathrm{bp}$ & $56^{\circ} \mathrm{C}$ & [18] \\
\hline & TTCTTCCGATTGACGCAAAA & & & \\
\hline \multirow[t]{2}{*}{ ace } & CAGGCCAACATCAAGCAACA & $125 \mathrm{bp}$ & $58^{\circ} \mathrm{C}$ & [18] \\
\hline & GCTTGCCTCGCCTTCTACAA & & & \\
\hline \multirow[t]{2}{*}{ gele } & CGAAGTTGGAAAAGGAGGC & $372 \mathrm{bp}$ & $54^{\circ} \mathrm{C}$ & [18] \\
\hline & GGTGAAGAAGTTACTCTGA & & & \\
\hline \multirow[t]{2}{*}{ hyl } & ACAGAAGAGCTGCAGGAAATG & $276 \mathrm{bp}$ & $55^{\circ} \mathrm{C}$ & [19] \\
\hline & GACTGACGTCCAAGTTTCCAA & & & \\
\hline \multirow[t]{2}{*}{ Cy/A } & ACTCGGGGATTGATAGGC & $688 \mathrm{bp}$ & $56^{\circ} \mathrm{C}$ & [19] \\
\hline & GCTGCTAAAGCTGCGCTT & & & \\
\hline \multirow[t]{2}{*}{ esp } & TTGGGGCAACTGGAATAGT & $407 \mathrm{bp}$ & $56^{\circ} \mathrm{C}$ & [18] \\
\hline & CCCAGCAAATAGTCCATCAT & & & \\
\hline
\end{tabular}

and ciprofloxacin $(5 \mu \mathrm{g})$ (MAST, Merseyside, U.K) was assessed according to the Clinical and Laboratory Standards Institute guidelines (CLSI) [20]. Multidrug resistance was defined as resistance to three or more different classes of antibiotics. Minimum inhibitory concentration (MIC) of vancomycin was determined using the agar dilution method according to CLSI guidelines [20]. The MIC was recorded as the lowest concentration that 
completely inhibited growth except for a single colony or a faint haze caused by the inoculum. Enterococcus faecalis ATCC29212 was used as reference strain for susceptibility testing.

\section{DNA extraction}

Enterococcal DNA was extracted by suspending a loop of overnight colonies in a tube containing $100 \mu \mathrm{l} \mathrm{TE}$ buffer (10 mM Tris-HCl; 1 mM EDTA, pH 8.0) (Merck, Germany) and $0.5 \mu$ lysozyme $(100 \mathrm{mg} / \mathrm{ml})$ (Sigma-Aldrich, USA), and incubated at $37^{\circ} \mathrm{C}$ for $1 \mathrm{~h}$. The suspensions boiled for $10 \mathrm{~min}$ and centrifuged at $14,000 \mathrm{rpm}$ for $5 \mathrm{~min}$ at room temperature [11]. The supernatants were collected and stored at $-20^{\circ} \mathrm{C}$ as DNA template stocks. The concentration and purity of DNA samples were determined using a NanoDrop Spectrophotometer (ND-1000, Nano-Drop Technologies, Wilmington, DE) at 260 and $260 / 280 \mathrm{~nm}$, respectively.

\section{Detection of resistance and virulence genes}

The presence of vancomycin resistance genes $v a n A$, vanB, vanC, aminoglycoside resistance genes aac (6')-Ieaph (2“)-Ia, aph (3')-IIIa, ant (4')-Ia, aph (2")-Ic, aph (2")-Ib, aph (2")-Id, ant (3")-III, ant (6')-Ia and virulence genes gelE, PAI, esp, ace, cyl, hyl and sprE was assessed using PCR method (The primers [Metabion, Germany] are shown in Table 1) [21-27]. Polymerase chain reaction was performed using DreamTaq PCR Master Mix (Ampliqon, Denmark), which contains Taq polymerase, dNTPs, $\mathrm{MgCl} 2$ and the appropriate buffer. Each PCR tube contained $25 \mu \mathrm{l}$ reaction mixture composed of $12.5 \mu \mathrm{l}$ of the master mix, $1.5 \mu \mathrm{l}$ of each forward and reverse primer solution (in a final concentration of 200 $\mathrm{nM}), 5 \mu \mathrm{l}$ of DNA with concentration of $100 \mathrm{ng} / \mu \mathrm{l}$ and nuclease-free water to complete the final volume. Amplification was performed using the Gene Atlas 322 system (ASTEC, Japan) with initial denaturation at $94^{\circ} \mathrm{C}, 5 \mathrm{~min}$ followed by 30 cycles of denaturation $\left(94^{\circ} \mathrm{C}, 1 \mathrm{~min}\right)$, annealing $\left(54-58^{\circ} \mathrm{C}, 45 \mathrm{~s}\right)$ and extension $\left(72^{\circ} \mathrm{C}, 1 \mathrm{~min}\right)$, with a final extension step $\left(72{ }^{\circ} \mathrm{C}, 10 \mathrm{~min}\right)$. The amplified DNA was separated by submarine gel electrophoresis, stained with ethidium bromide and visualized under UV transillumination (UVITEC, UK). Enterococcus faecalis ATCC 51299 and E. faecalis MMH594 was used as the positive control strain.

\section{Phenotypic detection of virulence factors Cytolysin activity}

Cytolysin activity was assessed on Brain Heart Infusion (BHI) Agar (Merck, Germany) supplemented with 5\% horse blood. Cytolytic activity was detected after $24 \mathrm{~h}$ incubation at $37^{\circ} \mathrm{C}$ as $\beta$-hemolysis surrounding bacterial colonies [28]. All assays were performed in triplicate.

\section{Gelatinase activity}

Gelatinase activity was assessed using $3 \%$ gelatin medium (Merck, Germany) as described previousely [12]. All assays were performed in triplicate.

\section{Haemagglutination assay}

Haemagglutination assay was performed according to Elsner et al. [12]. Enterococcal isolates were incubated for $24 \mathrm{~h}$ at $37^{\circ} \mathrm{C}$ on BHI Agar (Merck, Germany) supplemented with $10 \%$ sheep blood. Bacterial suspension with final concentration of $1.8 \times 10^{9} \mathrm{CFU} / \mathrm{mL}$ was prepared in phosphate-buffered saline (PBS). Then, $50 \mu 1$ of bacterial suspension mixed gently with $50 \mu \mathrm{l}$ of $3 \%$ human erythrocyte (collected from healthy volunteer people) suspension in PBS (pH 7.4) in 96-well U-bottom microtiter plates. Haemagglutination was recorded after rotating the plates for $5 \mathrm{~min}$ and then keeping them at room temperature for $30 \mathrm{~min}$. All assays were performed in triplicate.

\section{Biofilm forming assay}

Biofilm forming capacity was determined using microtiter plate as described by Zeighami et al. [29]. Biofilm formation was scored as follows:, non-biofilm forming (A595<1); + , weak $(1<$ A595 $\leq 2) ;++$, moderate $(2<$ A595 $\leq 3$ ); +++ , strong (A595 $>3$ ). Reported values are the mean of three measurements.

\section{Statistical analysis}

The data were analyzed with SPSS version 17.0 software (SPSS, Inc., Chicago, IL). A chi-square and Fisher s Exact tests were used to determine the statistical significance of the data. A $P$ value of $<0.05$ was considered significant.

\section{Results}

\section{Patient demographics}

A total of 100 enterococci were collected from urine samples of hospitalized patients with symptomatic UTI. Among the total patients, $48(48 \%)$ patients were younger than 30 years, 36 (36\%) were $30-45$ years and $16(16 \%)$ were $>45$ years. The sex distribution was $64(64 \%)$ female and $36(36 \%)$ male. Of 100 enterococci, 69 isolates were identified as E. faecalis, 10 isolates as E. faecium and 21 isolates as "other" Enterococcus species.

\section{Antimicrobial susceptibility}

Antibiotic resistance profile of isolates is presented in Table 2. Overall, 93 isolates were resistant to one or more antimicrobial agents, with the most frequent resistance found against tetracycline (86\%), ciprofloxacin (73\%) and quinupristin-dalfopristin (53\%). Gentamicin and streptomycin resistance was detected in 50 and 34\% 
Table 2 Antimicrobial resistance of Enterococcus species

\begin{tabular}{lllll}
\hline Antimicrobial agents & $\begin{array}{l}\text { No. (\%) of } \\
\text { resistant } \\
\text { E. faecalis } \\
(n=69)\end{array}$ & $\begin{array}{l}\text { Nosistant } \\
\text { E. faecium } \\
(n=10)\end{array}$ & $\begin{array}{l}\text { No. (\%) of } \\
\text { Enterococcal } \\
\text { spp. }(n=21)\end{array}$ & $\begin{array}{l}\text { No. (\%) of } \\
\text { total } \\
\text { resistant } \\
\text { isolates } \\
(n=100)\end{array}$ \\
\hline $\begin{array}{l}\text { Vancomycin } \\
\text { Ampicillin }\end{array}$ & $0(0)$ & $7(70)$ & $14(66.6)$ & $21(21)$ \\
Tetracycline & $0(0)$ & $10(100)$ & $12(57.1)$ & $22(22)$ \\
Gentamicin & $31(45)$ & $7(70)$ & $12(57.1)$ & $50(50)$ \\
Linezolid & $4(5.8)$ & 0 & $1(4.76)$ & $5(5)$ \\
Ciprofloxacin & $47(68.1)$ & $9(90)$ & $17(80.9)$ & $73(73)$ \\
Chloramphenicol & $16(23.2)$ & $2(20)$ & $3(14.3)$ & $21(21)$ \\
Fosfomycin & $1(1.44)$ & $0(0)$ & $0(0)$ & $1(1)$ \\
Quinupristin- & $34(34.8)$ & $6(60)$ & $13(61.9)$ & $53(53)$ \\
Dalfopristin & & & & \\
Streptomycin & $19(27.5)$ & $5(50)$ & $10(47.6)$ & $34(34)$ \\
\hline
\end{tabular}

of isolates, respectively. Fosfomycin showed the highest activity against isolates and only one isolate was fosfomycin resistant. Furthermore, $95 \%$ of isolates were susceptible to linezolid.

Twenty one isolates were resistant to vancomycin, with $\mathrm{MICs} \geq 32 \mu \mathrm{g} / \mathrm{ml}$, and $15 \%$ of isolates showed MIC of vancomycin $\geq 256 \mu \mathrm{g} / \mathrm{ml}$ and considered as high level vancomycin resistant (HLVR) [30]. Of 21 vancomycin resistant enterococci, 10 isolates were identified as $E$. faecium and 11 isolates as "other" Enterococcus species. No ampicillin or vancomycin resistant $E$. faecalis isolate was detected.

A total of 36 isolates were resistant to at least three different classes of antimicrobial agents and considered as MDR. The most prevalent MDR pattern was resistance to tetracycline, ciprofloxacin, gentamicin and quinupristin-dalfopristin.

\section{Distribution of vancomycin and aminoglycoside} resistance genes

Among 21 VRE isolates, 12 (57.1\%) isolates were positive for the presence of van genes. Ten E. faecium (47.6\%) isolates carried vanA and 2 (9.5\%) Enterococcus species carried vanC.

The distribution of aminoglycoside resistance genes (ARGs) is presented in Table 3 . The most prevalent ARG was ant (3')-III (78\%), followed by aph (3')-IIIa (67\%), ant (6')Ia (62\%) and aac (6')-Ie-aph (2")-Ia (15\%). The frequency of aph (2")-Ib and ant (4')-Ia was 7 and $4 \%$, respectively. Frequency of ARGs in E. faecium and $E$. faecalis isolates did not show significant difference.

The presence of multiple ARGs with different combinations was found in enterococci. Eighty percent of $E$. faecium and $78.3 \%$ of $E$. faecalis isolates were carried two or more ARGs (Table 4). The number of ARGs per isolate and their specific combinations are shown in Table 4. The most frequent combinations of ARGs in enterococci were ant (6')Ia + ant (3')-IIIa + aph (3')-IIIa (15.2\%), followed by aph (3')-IIIa + ant (3')-III (12.6\%) and ant (6')Ia + aph (3')-IIIa (10.1\%).

\section{Distribution of enterococcal virulence related genes}

The virulence related genes PAI, sprE, ace, gelE, hyl, cylA and espE were detected in E. faecalis and E. faecium isolates. The frequency of enterococcal virulence genes is shown in Table 5. The most frequent virulence gene was ace (88.6\%), followed by esp (67.1\%), PAI (45.5\%) and sprE (41.7\%). As shown in Table 5, the frequency of ace, cylA and esp genes among $E$. faecalis isolates was significantly higher than $E$. faecium $(P<0.05)$. All $E$. faecalis isolates carried at least one virulence gene. However, gelE, and cylA genes were not detected in E. faecium isolates.

Several different combinations of virulence genes were found in enterococci. Table 6 shows that 97.1\% of $E$.

Table 3 Frequency of vancomycin and aminoglycoside resistance genes in Enterococcus species

\begin{tabular}{|c|c|c|c|c|}
\hline Genes & $\begin{array}{l}\text { No. (\%) of } \\
\text { E. faecalis }(n=69)\end{array}$ & $\begin{array}{l}\text { No. }(\%) \text { of } \\
\text { E. faecium }(n=10)\end{array}$ & $\begin{array}{l}\text { No. }(\%) \text { of } \\
\text { Other species } \\
(n=21)\end{array}$ & $\begin{array}{l}\text { No. (\%) of Total } \\
(n=100)\end{array}$ \\
\hline$a a c\left(6^{\prime}\right)-1 e-a p h\left(2^{\prime \prime}\right)-1 a$ & $10(14.5)$ & $1(10)$ & $4(19)$ & $15(15)$ \\
\hline$a p h\left(3^{\prime}\right)-I I I a$ & $46(66.6)$ & $7(70)$ & $14(66.7)$ & $67(67)$ \\
\hline $\operatorname{ant}\left(4^{\prime}\right)-1 a$ & $3(4.3)$ & $0(0)$ & $1(4.7)$ & $4(4)$ \\
\hline $\operatorname{aph}\left(2^{\prime \prime}\right)-/ c$ & $6(8.7)$ & $0(0)$ & $2(9.5)$ & $8(8)$ \\
\hline$a p h\left(2^{\prime \prime}\right)-1 b$ & $4(5.8)$ & $2(20)$ & $1(4.7)$ & $7(7)$ \\
\hline $\operatorname{aph}\left(2^{\prime \prime}\right)-1 d$ & $5(7.2)$ & $2(20)$ & $1(4.7)$ & $8(8)$ \\
\hline $\operatorname{ant}\left(3^{\prime \prime}\right)-$ III & $50(72.5)$ & $9(90)$ & $19(90.5)$ & $78(78)$ \\
\hline$a n t\left(6^{\prime}\right)-l a$ & $41(59.4)$ & $6(60)$ & $15(71.4)$ & $62(62)$ \\
\hline $\operatorname{van} A$ & 0 & $10(100)$ & 0 & $10(10)$ \\
\hline $\operatorname{van} B$ & 0 & 0 & 0 & 0 \\
\hline $\operatorname{van} C$ & 0 & 0 & $2(9.5)$ & $2(2)$ \\
\hline
\end{tabular}


Table 4 Frequency of different combinations of ARG among Enterococcus species

\begin{tabular}{|c|c|c|c|c|}
\hline No. of ARG & ARG combinations & $\begin{array}{l}\text { No. (\%) of ARGs in E. faecalis } \\
(n=69)\end{array}$ & $\begin{array}{l}\text { No. (\%) of ARGs in E. faecium } \\
(n=10)\end{array}$ & $\begin{array}{l}\text { Total No. }(\%) \\
(n=79)\end{array}$ \\
\hline $\begin{array}{l}\text { Without } \\
\text { ARG }\end{array}$ & - & $1(1.4)$ & - & $1(1.2)$ \\
\hline \multirow[t]{3}{*}{$1 \mathrm{ARG}$} & $a n t\left(6^{\prime}\right) \mid a$ & $5(7.2)$ & - & $16(20.2)$ \\
\hline & $\operatorname{ant}\left(3^{\prime}\right)-111 a$ & $7(10.1)$ & $2(20)$ & \\
\hline & $a p h\left(3^{\prime}\right)-111 a$ & $2(2.8)$ & - & \\
\hline \multirow[t]{6}{*}{2 ARGs } & $a p h\left(3^{\prime}\right)--I I a+\operatorname{ant}\left(3^{\prime}\right)-I \| !$ & $8(11.5)$ & $2(20)$ & $26(32.9)$ \\
\hline & $\operatorname{ant}\left(6^{\prime}\right) \mid a+\operatorname{ant}\left(3^{\prime}\right)-I I I a$ & $4(5.7)$ & - & \\
\hline & $\operatorname{ant}\left(6^{\prime}\right) \mid a+a p h\left(3^{\prime}\right)-I \| a$ & $8(11.5)$ & - & \\
\hline & $\operatorname{ant}\left(3^{\prime}\right)-111 a+a a c\left(6^{\prime}\right)-l e$ & $1(1.4)$ & - & \\
\hline & $\operatorname{ant}\left(3^{\prime}\right)-1\left\|l a+a p h\left(3^{\prime}\right)-1\right\| I a$ & $2(2.8)$ & - & \\
\hline & $\operatorname{ant}\left(6^{\prime}\right) \mid a+a a c\left(6^{\prime}\right)-l e$ & $1(1.4)$ & - & \\
\hline \multirow[t]{10}{*}{3 ARGs } & $\operatorname{ant}\left(3^{\prime}\right)-I I\left|a+a p h\left(2^{\prime \prime}\right)-\right| c+\operatorname{ant}\left(4^{\prime}\right) \mid a$ & $1(1.4)$ & - & $22(27.8)$ \\
\hline & $\operatorname{ant}\left(6^{\prime}\right) \mid a+\operatorname{ant}\left(3^{\prime}\right)-\|\| a+a p h\left(3^{\prime}\right)-\|\| a$ & $10(14.4)$ & $2(20)$ & \\
\hline & $\operatorname{ant}\left(6^{\prime}\right) \mid a+a p h\left(3^{\prime}\right)-1 \| a+a p h\left(2^{\prime \prime}\right)-1 c$ & $1(1.4)$ & - & \\
\hline & $\operatorname{ant}\left(3^{\prime}\right)-\left|\left\|\left|a+a p h\left(3^{\prime}\right)-\right|\right\| a+\operatorname{ant}\left(4^{\prime}\right)\right| a$ & $1(1.4)$ & - & \\
\hline & $\operatorname{ant}\left(3^{\prime}\right)-I I I a+a p h\left(3^{\prime}\right)-I I a+a p h\left(2^{\prime \prime}\right)-1 c$ & $1(1.4)$ & - & \\
\hline & $a n t\left(3^{\prime}\right)-11 l a+a p h\left(3^{\prime}\right)-111 a+a a c\left(6^{\prime}\right)-l e$ & $1(1.4)$ & - & \\
\hline & $\operatorname{ant}\left(6^{\prime}\right) \mid a+a a c\left(6^{\prime}\right)-l e+a p h\left(2^{\prime \prime}\right)-l c$ & $1(1.4)$ & - & \\
\hline & $\operatorname{ant}\left(6^{\prime}\right) \mid a+\operatorname{ant}\left(3^{\prime}\right)-11 a+\operatorname{aph}\left(2^{\prime \prime}\right)-1 b$ & $1(1.4)$ & $1(10)$ & \\
\hline & $\operatorname{ant}\left(6^{\prime}\right)\left|a+a p h\left(3^{\prime}\right)-1\right| l a+a a c\left(6^{\prime}\right)-l e$ & - & $1(10)$ & \\
\hline & $\operatorname{ant}\left(3^{\prime}\right)-I I I a+a p h\left(2^{\prime \prime}\right)-I d+a p h\left(3^{\prime}\right)-I I I a$ & $1(1.4)$ & - & \\
\hline \multirow[t]{7}{*}{4 ARGs } & $\operatorname{ant}\left(6^{\prime}\right)\left|a+\operatorname{ant}\left(3^{\prime}\right)-I I\right| a+a p h\left(2^{\prime \prime}\right)-I d+a p h\left(3^{\prime}\right)-I I I a$ & $2(2.8)$ & $1(10)$ & $12(15.1)$ \\
\hline & $\operatorname{ant}\left(6^{\prime}\right) \mid a+\operatorname{ant}\left(3^{\prime}\right)-I I I a+a p h\left(3^{\prime}\right)-I I I a+a a c\left(6^{\prime}\right)-l e$ & $4(5.7)$ & - & \\
\hline & $\operatorname{ant}\left(6^{\prime}\right)\left|a+\operatorname{ant}\left(3^{\prime}\right)-\left\|l\left|a+a p h\left(2^{\prime \prime}\right)-1 b+a p h\left(3^{\prime}\right)-\| l\right| a\right.\right.$ & $1(1.4)$ & - & \\
\hline & $\operatorname{ant}\left(3^{\prime}\right)-\left\|I I a+a p h\left(2^{\prime \prime}\right)-I d+a p h\left(3^{\prime}\right)-\right\| I a+a p h\left(2^{\prime \prime}\right)-1 c$ & $1(1.4)$ & - & \\
\hline & $\operatorname{ant}\left(6^{\prime}\right)\left|a+\operatorname{ant}\left(3^{\prime}\right)-\| l\right| a+a p h\left(2^{\prime \prime}\right)-l d+a a c\left(6^{\prime}\right)-l e$ & $1(1.4)$ & - & \\
\hline & $\operatorname{ant}\left(6^{\prime}\right)\left|a+\operatorname{ant}\left(3^{\prime}\right)-\left\|1\left|a+a p h\left(3^{\prime}\right)-\| I a+a p h\left(2^{\prime \prime}\right)-\right| c\right.\right.$ & $1(1.4)$ & - & \\
\hline & $\operatorname{ant}\left(3^{\prime}\right)-1 \| a+a p h\left(2^{\prime \prime}\right)-\left|b+a p h\left(3^{\prime}\right)-\right| I\left|a+\operatorname{ant}\left(4^{\prime}\right)\right| a$ & $1(1.4)$ & - & \\
\hline \multirow[t]{2}{*}{5 ARGS } & $\begin{array}{l}a n t\left(6^{\prime}\right)\left|a+a n t\left(3^{\prime}\right)-11\right| a+a p h\left(2^{\prime \prime}\right)-1 b+a p h\left(3^{\prime}\right)-11 l a+a a c \\
\left(6^{\prime}\right)-l e\end{array}$ & $1(1.4)$ & - & $2(2.5)$ \\
\hline & $\begin{array}{l}\operatorname{ant}\left(6^{\prime}\right) \mid a+\operatorname{ant}\left(3^{\prime}\right)-I I a+a p h\left(2^{\prime \prime}\right)-1 b+a p h\left(2^{\prime \prime}\right)-l d+ \\
a p h\left(3^{\prime}\right)-\| I \mid a\end{array}$ & - & $1(10)$ & \\
\hline
\end{tabular}

faecalis and $50 \%$ of E. faecium isolates harbored two or more virulence genes simultaneously $(P<0.05)$. The mean number of virulence genes per isolate was higher in E. faecalis isolates than E. faecium $(P<0.05)$. The most frequent combination in E. faecalis was PAI-sprEace-esp (13\%), followed byPAI-ace-cylA-esp and acecylA-esp (8.6\%).

\section{Phenotypic detection of virulence factors}

Gelatinase and cytolytic activity, haemagglutination and biofilm formation was assayed by phenotypic tests (Table 7). Hemolytic activity of $E$. faecalis isolates (46.4\%) was higher than other Enterococcus spp. (14.3\%). Hemolytic activity cannot be detected in E. faecium isolates. Gelatinase activity was detected in $25 \%$ of enterococci. However, there was no significant difference among Enterococcus species in gelatinase activity $(P>0.05)$. Haemagglutination and biofilm formation phenotypes were detected in 75 and $74 \%$ of enterococci. Biofilm formation in E. faecalis isolates (89.8\%) was significantly higher than other species. Of 74 biofilm forming isolates, 64 isolates produced weak biofilm $(+)$ and 10 isolates formed moderate biofilm $(++)$. Frequency of resistance genes among virulence factors producing entrococci is shown in Table 8. The resistance genes of aph (3')IIIa, ant (3')-III and ant (6')Ia were detected significantly higher among enterococci with haemagglutination and biofilm formation phenotypes $(P<0.05)$. Also, all resistance genes (except vanC, ant (4')Ia and aph (3')-IIIa) were 
Table 5 Frequency of virulence genes among Enterococcus

\begin{tabular}{lllll}
$\begin{array}{l}\text { species } \\
\text { virulence } \\
\text { genes }\end{array}$ & $\begin{array}{l}\text { E. faecalis } \\
(n=69)\end{array}$ & $\begin{array}{l}\text { E. faecium } \\
(n=10)\end{array}$ & $P$ value & $\begin{array}{l}\text { Total } \\
(n=79)\end{array}$ \\
\hline PAl & $31(44.9)$ & $5(50)$ & 0.488 & $36(45.5)$ \\
sprE & $30(43.4)$ & $3(30)$ & 0.142 & $33(41.7)$ \\
ace & $62(89.8)$ & $8(80)$ & $0.001^{*}$ & $70(88.6)$ \\
gelE & $17(24.6)$ & 0 & 0.063 & $17(21.5)$ \\
hyl & $2(2.8)$ & $1(10)$ & 0.337 & $3(3.8)$ \\
cylA & $25(36.2)$ & 0 & $0.017^{*}$ & $25(31.6)$ \\
esp & $50(72.4)$ & $3(30)$ & $0.012^{*}$ & $53(67.1)$
\end{tabular}

Fisher $\mathrm{s}$ Exact test was used to determine the statistical significance of the data

${ }^{*} P$ value of $<0.05$ was considered significant

Table 6 Frequency of different combinations of virulence genes among Enterococcus species

\begin{tabular}{|c|c|c|}
\hline Genetic profile & $\begin{array}{l}\text { No. (\%) of E. faecalis } \\
(n=69)\end{array}$ & $\begin{array}{l}\text { No. }(\%) \text { of } E \text {. faecium } \\
(n=10)\end{array}$ \\
\hline No virulence factor & - & - \\
\hline PAl & - & $1(10)$ \\
\hline Ace & $2(2.8)$ & $4(40)$ \\
\hline PAl-ace & $1(1.4)$ & - \\
\hline spre-ace & $2(2.8)$ & - \\
\hline ace-gelE & $1(1.4)$ & - \\
\hline ace-hyl & $1(1.4)$ & $1(10)$ \\
\hline PAl-esp & $1(1.4)$ & $1(10)$ \\
\hline ace-esp & $4(5.7)$ & - \\
\hline gelE-esp & $2(2.8)$ & - \\
\hline cylA-esp & $1(1.4)$ & - \\
\hline PAl-sprE-ace & $4(5.7)$ & $1(10)$ \\
\hline sprE-ace-gele & $3(4.3)$ & - \\
\hline PAl-ace-cylA & $1(1.4)$ & - \\
\hline ace-gelE-cylA & $1(1.4)$ & - \\
\hline ace-hyl-cylA & $1(1.4)$ & - \\
\hline PAl-sprE-esp & $2(2.8)$ & - \\
\hline PAl-ace-esp & $5(7.2)$ & - \\
\hline sprE-ace-esp & $4(5.7)$ & - \\
\hline ace-gelE-esp & $2(2.8)$ & - \\
\hline ace-cylA-esp & $6(8.6)$ & - \\
\hline sprE-ace-gelE-cy|A & $2(2.8)$ & - \\
\hline PAl-sprE-ace-esp & $9(13)$ & $2(20)$ \\
\hline sprE-ace-gelE-esp & $1(1.4)$ & - \\
\hline PAl-ace-cylA-esp & $6(8.6)$ & - \\
\hline sprE-gelE-cy|A-esp & $1(1.4)$ & - \\
\hline ace-gelE-cylA-esp & $4(5.7)$ & - \\
\hline PAl-sprE-ace-cylA-esp & $2(2.8)$ & - \\
\hline
\end{tabular}

frequently detected in isolates with gelatinase and cytolytic activity $(\mathrm{P}<0.05)$.

\section{Discussion}

Multidrug resistant enterococci, as important nosocomial pathogens, have become a serious problem in hospitalized patients $[3,6]$. Due to the extensive misuse of antimicrobial agents in our country, treatment of infections associated with MDR enterococci is complicated $[31,32]$. In our study, $93 \%$ of enterococci were resistant to one or more antimicrobial agents and $36 \%$ were MDR. The frequency of antimicrobial resistance among E. faecium isolates was more than E. faecalis (except for linezolid and fosfomycin). The inherent antibiotic resistance and dissemination of resistance genes through conjugative transposons and plasmids play an important role in development of MDR enterococci [33]. High frequency of antimicrobial resistance among enterococci was reported in previous studies from Iran [3, 32, 34].

While intrinsic mechanisms result in low level aminoglycoside resistance, acquisition of mobile genetic elements typically underlies high level aminoglycoside resistance in E. faecium and E. faecalis [4]. High level aminoglycoside resistance among enterococci was first reported in France in 1979 and since then has caused serious problems in healthcare settings worldwide [6]. Recent studies indicated that HLGR among enterococci to be more common than HLSR [35]. Similarly, gentamycin resistant enterococci $(50 \%)$ were detected with higher frequency than streptomycin resistance (34\%) in our study. Also, Mirnejad et al. and Zarrilli et al. were reported that 56.9 and $46.1 \%$ of enterococci were HLGR, respectively $[9,13]$.

Aminoglycoside resistance genes in E. faecium isolates were detected with higher frequency than E. faecalis, an observation which is consistent with that found in previous reports $[6,35]$. While previous studies found that aac (6')-Ie-aph (2")-Ia was the most common ARG [6, 9, 35], we detected aac (6')-Ie-aph (2")-Ia with lower frequency in $15 \%$ of enterococci. According to our results, the most prevalent ARG was ant (3")-III (78\%), followed by aph (3')-IIIa (67\%), ant (6')Ia (62\%).

Furthermore, $80 \%$ of E. faecium and $78.3 \%$ of E. faecalis isolates were carried two or more ARGs. Our results are consistent with previous reports on the predominance of enterococci with two or more ARGs [9, 35] .

High level vancomycin resistance, leading causes of hospital-acquired infections, were first reported in United Kingdom in 1980s and since then have caused significant public health concern because of its propensity to acquire and transfer the mobile resistance genes [14]. As reported in previous studies, the most common risk factors for VRE infections are prolonged hospitalization, use of vancomycin and third-generation cephalosporins and chronic dialysis [15]. In 
Table 7 Phenotypic detection of virulence factors in Enterococcus species

\begin{tabular}{llllll}
\hline $\begin{array}{l}\text { Virulence } \\
\text { factors }\end{array}$ & $\begin{array}{l}\text { E. faecalis } \\
(n=69)\end{array}$ & $\begin{array}{l}\text { E. faecium } \\
(n=10)\end{array}$ & $\begin{array}{l}\text { Other spp. } \\
(n=21)\end{array}$ & $P$ value & $\begin{array}{l}\text { Total } \\
(n=100)\end{array}$ \\
& No. (\%) & No. (\%) & No. (\%) & & No. (\%) \\
\hline Cytolytic activity & $32(46.4)$ & 0 & $3(14.3)$ & $0.001^{*}$ & $35(35)$ \\
Gelatinase activity & $18(26.1)$ & 0 & $7(33.3)$ & 0.107 & $25(25)$ \\
Hemagglutination & $55(79.7)$ & $7(70)$ & $13(61.9)$ & 0.245 & $75(75)$ \\
Biofilm formation & $62(89.8)$ & $2(20)$ & $10(47.6)$ & $0.000^{*}$ & $74(74)$
\end{tabular}

Fisher $\mathrm{s}$ Exact test was used to determine the statistical significance of the data

${ }^{*} P$ value of $<0.05$ was considered significant

our study, $15 \%$ of enterococci were high level vancomycinresistant with $\mathrm{MIC}$ of $\geq 256 \mu \mathrm{g} / \mathrm{ml}$ and $21 \%$ were vancomycin resistant which consistent with some previous reports $[15,16]$. No vancomycin resistant $E$. faecalis $\left(\operatorname{VRE}_{f s}\right)$ was found in our study. In contrast to our results, the frequency of $\mathrm{VRE}_{f s}$ was higher than vancomycin resistant E. faecium $\left(\mathrm{VRE}_{f m}\right)$ in study carried out by Sabouni et al. in Iran [16]. Similar to our study, Wisplinghoff et al. reported vancomycin resistance in $2 \%$ of $E$. faecalis and $60 \%$ of E. faecium isolates. However, they did not report high level vancomycin resistance [17]. National survey data have indicated the prevalence of VRE in $0-59 \%$ of isolates in 126 adult ICUs from 60 US hospitals [18]. Several reports also showed the elevated occurrence of $v a n A$ in comparison to other $v a n$ types $[5,16,17]$. We found a high occurrence of vanA in VRE isolates. Several hospitals located in São Paulo and other Brazilian cities reported both outbreaks and isolated cases of VRE infection/colonization [19]. All VRE $\mathrm{V}_{f m}$ isolates carried $v a n A$ but $v a n B$ was not detected among enterococci in our study. Similar to our results, Cekin et al. did not detected $v a n B$ among enterococci [31].

The essential virulence factors for pathogenicity of enterococci have not yet been described and the pathogenicity has been considered a multifactorial process [10]. Previous studies showed the association between the presence of virulence factors and promoting emergence of enterococcal infections in nosocomial settings $[5,10]$. Our results showed different prevalence of virulence genes in enterococci which ranged from 3.9 to $8.6 \%$. The most frequent virulence genes were ace and esp. Ace is an adhesion of collagen from Enterococcus that binds to collagen and laminin and belongs to the MSCRAMM family. In Bulgaria, Strateva et al. reported varied distribution of esp in non-invasive E. faecalis isolates (54.3$64.8 \%$ ) compared to invasive isolates (33.3\%) [2]. In our study, ace and esp were found respectively among 88.6 and $67.1 \%$ of enterococci isolated from UTIs, which confirm the important role of Ace and Esp as colonization factors in UTIs. The frequency of ace and esp in E. faecalis isolates was significantly higher than E. faecium. A strong correlation between the presence of Esp and the ability of an Enterococcus isolate to colonizes and persists in urinary tract and forms biofilm in vitro has been reported [10]. According to our results, 74\% of enterococci showed biofilm formation phenotype which exhibits an important role of biofilm formation in UTIs. The virulence genes gelE and $c y l A$ were not detected in E. faecium isolates. Similar to our results, a multicenter study on distribution of virulence determinants in fecal E. faecium isolates of patients in 13 hospitals from nine European countries showed total absence of gelE gene. However, 26 and $36.2 \%$ of E. faecalis isolates carried gelE and $c y l A$ determinants, respectively. The least prevalence among enterococci was $h y l$ which was detected in only 3 isolates (3.9\%). Similar to our results, Soheili et al. demonstrated that only $8 \%$ of E. faecalis isolates in Malaysian patients carried $h y l$ [1]. Since $h y l$ was not prevalent in our study and some previous reports [1, 28], we believe that this gene could has little role in pathogenicity of Enterococcus in comparison with other prevalent virulence genes.

According to our results, $97.1 \%$ of E. faecalis and 50\% of $E$. faecium isolates harbored two or more virulence genes simultaneously $(P<0.05)$. Furthermore, among gentamycin resistant enterococci, 45 isolates $(90 \%)$ were carried at least two or more virulence genes which is consistent with previous reports [2, 6, 35]. Also, 42\% of VRE isolates were harbored at least two or more virulence determinants.

One of the limitations of our study was the low number of E. faecium isolates and since the study was conducted in small geographical area, Northwest of Iran, the results cannot be generalized. For better characterization of enterococci

Table 8 Frequency of resistance genes among virulence factors producing entrococci

\begin{tabular}{|c|c|c|c|c|c|c|c|c|c|c|}
\hline $\begin{array}{l}\text { Resistance genes } \\
\text { Virulence factors }\end{array}$ & $\begin{array}{l}\operatorname{Van} A \\
(n=10)\end{array}$ & $\begin{array}{l}\operatorname{Van} C \\
(n=2)\end{array}$ & $\begin{array}{l}a a c\left(6^{\prime}\right)-l e-a p h \\
\left(2^{\prime \prime}\right)-l a(n=20)\end{array}$ & $\begin{array}{l}\text { aph(3')-IIIa } \\
(n=71)\end{array}$ & $\begin{array}{l}\text { ant }\left(4^{\prime}\right)-1 a \\
(n=6)\end{array}$ & $\begin{array}{l}\text { aph }\left(2^{\prime \prime}\right)-/ c \\
(n=9)\end{array}$ & $\begin{array}{l}\text { aph }\left(2^{\prime \prime}\right)-1 b \\
(n=5)\end{array}$ & $\begin{array}{l}\text { aph(2")-Id } \\
(n=7)\end{array}$ & $\begin{array}{l}\operatorname{ant}\left(3^{\prime \prime}\right)-I I I \\
(n=84)\end{array}$ & $\begin{array}{l}\text { ant }\left(6^{\prime}\right)-1 a \\
(n=57)\end{array}$ \\
\hline Cytolytic activity $(n=35)$ & $8^{*}$ & 1 & $14^{*}$ & $20^{*}$ & 4 & $8^{*}$ & $4^{*}$ & $6^{*}$ & $34^{*}$ & $32^{*}$ \\
\hline Gelatinase activity $(n=25)$ & $8^{*}$ & 2 & $18^{*}$ & 16 & $6^{*}$ & $9^{*}$ & $4^{*}$ & $7^{*}$ & $25^{*}$ & $25^{*}$ \\
\hline Hemagglutination $(n=75)$ & 7 & 1 & 13 & $60^{*}$ & 5 & 9 & 5 & 7 & $74^{*}$ & $53^{*}$ \\
\hline Biofilm formation $(n=74)$ & 10 & 1 & 17 & $66^{*}$ & 6 & 8 & 5 & 7 & $74^{*}$ & $56^{*}$ \\
\hline
\end{tabular}

Fisher $\mathrm{s}$ Exact test was used to determine the statistical significance of the data

${ }^{*} P$ value of $<0.05$ was considered significant 
strains, we suggest that molecular typing methods such as pulsed-field gel electrophoresis and multilocus sequence typing will be done.

\section{Conclusion}

Our study demonstrated that E. faecalis was more common than other Enterococcus species, but high frequency of aminoglycoside and vancomycin resistance was detected among $E$. faecium isolates. The distribution of virulence genes (except $h y l$ ) among $E$. faecalis isolates was higher than $E$. faecium. Due to high frequency of MDR enterococci, it seems that the appropriate surveillance and control measures are essential to prevent the emergence and transmission of these isolates in hospitals. Further studies should be carried out for a better understanding of the association between the presence of virulence determinants and emergence of multidrug resistant enterococci.

\section{Abbreviations}

AAC: Aminoglycoside acetyl transferase; AME: Aminoglycoside modifying enzyme; ANT: Aminoglycoside nucleotidyl transferase; APH: Aminoglycoside phosphoryl transferase; ARG: Aminoglycoside resistance gene; HLAR: High level aminoglycoside resistance; MDR: Multidrug resistant; PYR: L-Pyrrolidonyl$\beta$-Naphthylamide; UTI: Urinary tract infection; VRE: Vancomycin resistant enterococci

\section{Acknowledgements}

The authors would like to acknowledge Aidin Jahanshahi for his support in sampling.

\section{Authors' contributions}

$\mathrm{FH}$ and $\mathrm{HZ}$ involved in the project development, data management and manuscript writing. $\mathrm{VL}$ participated in project development, data analysis. All authors have read and approved the final version of manuscript.

\section{Funding}

This work was funded as an MSc thesis in Medical Microbiology by the Zanjan University of Medical Sciences (A-12-392-17, A-12-392-14). The study was designed, conducted, analyzed, and written by the authors without involvement of any commercial party.

\section{Availability of data and materials}

The datasets will not be available on a publically available website, but it may be possible to provide access to anonymized data. Anyone who wants to request the data can contact with Habib Zeighami, corresponding author.

\section{Ethics approval and consent to participate}

Ethical clearance was obtained from the Ethics Committee of Zanjan University of Medical Sceinces before conducting the study (IR.ZUMS.REC.1394.324, ZUMS.REC.1394.177). All participants provided written informed consent to participate in the studies.

\section{Consent for publication}

Not applicable.

\section{Competing interests}

The authors declare that they have no competing interests.

\section{Author details}

'Department of Microbiology, School of Medicine, Zanjan University of Medical Sciences, Zanjan, Iran. ${ }^{2}$ Zanjan Pharmaceutical Biotechnology Research Center, Zanjan University of Medical Sciences, Zanjan, Iran. ${ }^{3}$ Department of Microbiology, Iran University of Medical Sciences, Tehran, Iran.
Received: 24 June 2019 Accepted: 20 August 2019

Published online: 27 August 2019

\section{References}

1. Soheili S, Ghafourian S, Sekawi Z, Neela V, Sadeghifard N, Ramli R, Hamat RA. Wide distribution of virulence genes among Enterococcus faecium and Enterococcus faecalis clinical isolates. Sci World J. 2014; 2014:623174.

2. Strateva T, Atanasova D, Savov E, Petrova G, Mitov I. Incidence of virulence determinants in clinical Enterococcus faecalis and Enterococcus faecium isolates collected in Bulgaria. Braz J Infect Dis. 2016;20(2):127-33.

3. Sharifi Y, Hasani A, Ghotaslou R, Naghili B, Aghazadeh M, Milani M, Bazmany A. Virulence and antimicrobial resistance in enterococci isolated from urinary tract infections. Adv Pharm Bull. 2013;3(1):197-201.

4. Hollenbeck BL, Rice LB. Intrinsic and acquired resistance mechanisms in enterococcus. Virulence. 2012;3(5):421-33.

5. Nasaj M, Mousavi SM, Hosseini SM, Arabestani MR. Prevalence of virulence factors and vancomycin-resistant genes among Enterococcus faecalis and $\mathrm{E}$. faecium isolated from clinical specimens. Iran J Public Health. 2016;45(6): $806-13$

6. Li W, Li J, Wei Q, Hu Q, Lin X, Chen M, Ye R, Lv H. Characterization of aminoglycoside resistance and virulence genes among Enterococcus spp. isolated from a hospital in China. Int J Environ Res Public Health. 2015;12(3): 3014-25.

7. Lebreton F, Depardieu F, Bourdon N, Fines-Guyon M, Berger P, Camiade S, Leclercq R, Courvalin P, Cattoir V. D-ala-d-Ser VanN-type transferable vancomycin resistance in Enterococcus faecium. Antimicrob Agents Chemother. 2011:55(10):4606-12.

8. Yamane K, Wachino J, Doi Y, Kurokawa H, Arakawa Y. Global spread of multiple aminoglycoside resistance genes. Emerg Infect Dis. 2005; 11(6):951-3.

9. Mirnejad R, Sajjadi N, Masoumi Zavaryani S, Piranfar V, Hajihosseini M, Roshanfekr M. Identification of aminoglycoside resistance genes by triplex PCR in Enterococcus spp. isolated from ICUs. Infez Med. 2016;24(3):222-9.

10. Comerlato CB, Resende MC, Caierao J, d'Azevedo PA. Presence of virulence factors in Enterococcus faecalis and Enterococcus faecium susceptible and resistant to vancomycin. Mem Inst Oswaldo Cruz. 2013;108(5):590-5.

11. Ferguson DM, Talavera GN, Hernández LA, Weisberg SB, Ambrose RF, Jay JA Virulence genes among Enterococcus faecalis and Enterococcus faecium isolated from coastal beaches and human and nonhuman sources in Southern California and Puerto Rico. J Pathog. 2016:2016:3437214.

12. Elsner HA, Sobottka I, Mack D, Claussen M, Laufs R, Wirth R. Virulence factors of Enterococcus faecalis and Enterococcus faecium blood culture isolates. Eur J Clin Microbiol Infect Dis. 2000;19(1):39-42.

13. Zarrilli R, Tripodi MF, Di Popolo A, Fortunato R, Bagattini M, Crispino M, Florio A, Triassi M, Utili R. Molecular epidemiology of high-level aminoglycoside-resistant enterococci isolated from patients in a university hospital in southern Italy. J Antimicrob Chemother. 2005;56(5):827-35.

14. Rosenberg Goldstein RE, Micallef SA, Gibbs SG, George A, Claye E, Sapkota A, Joseph SW, Sapkota AR. Detection of vancomycin-resistant enterococci (VRE) at four U.S. wastewater treatment plants that provide effluent for reuse. Sci Total Environ. 2014;466-467:404-11.

15. Ghalandarzadeh Daryaei Z, Javadpour S, Kargar M. Frequency of vana \& vanb genes in vancomycin-resistant enterococci isolated from clinical specimens at shahid mohammadi hospitals bandar abbass. J Microbial World. 2013;6(1):23-33.

16. Sabouni F, Movahedi Z, Mahmoudi S, Pourakbari B, Keshavarz Valian S, Mamishi S. High frequency of vancomycin resistant Enterococcus faecalis in children: an alarming concern. J Prev Med Hyg. 2016;57(4):E201-4.

17. Wisplinghoff $H$, Bischoff $T$, Tallent SM, Seifert $H$, Wenzel RP, Edmond MB. Nosocomial bloodstream infections in US hospitals: analysis of 24,179 cases from a prospective nationwide surveillance study. Clin Infect Dis. 2004;39(3):309-17.

18. Fridkin SK, Edwards JR, Courval JM, Hill H, Tenover FC, Lawton R, Gaynes RP, McGowan JE Jr. The effect of vancomycin and third-generation cephalosporins on prevalence of vancomycin-resistant enterococci in $126 \mathrm{U}$. S. adult intensive care units. Ann Intern Med. 2001:135(3):175-83.

19. Maschieto A, Martinez R, Palazzo IC, Darini AL. Antimicrobial resistance of Enterococcus sp. isolated from the intestinal tract of patients from a university hospital in Brazil. Mem Inst Oswaldo Cruz. 2004;99(7):763-7. 
20. M100-S27. Clinical and Laboratory Standards Institute, Performance Standards for Antimicrobial Susceptibility Testing. 27th ed; 2017. p. 94-7.

21. Kariyama R, Mitsuhata R, Chow JW, Clewell DB, Kumon H. Simple and reliable multiplex PCR assay for surveillance isolates of vancomycin-resistant enterococci. J Clin Microbiol. 2000;38(8):3092-5.

22. Clark NC, Cooksey RC, Hill BC, Swenson JM, Tenover FC. Characterization of glycopeptide-resistant enterococci from U.S. hospitals. Antimicrob Agents Chemother. 1993;37(11):2311-7.

23. Emaneini M, Aligholi M, Aminshahi M. Characterization of glycopeptides, aminoglycosides and macrolide resistance among Enterococcus faecalis and Enterococcus faecium isolates from hospitals in Tehran. Pol J Microbiol. 2008;57(2):173-8.

24. Vakulenko SB, Donabedian SM, Voskresenskiy AM, Zervos MJ, Lerner SA, Chow JW. Multiplex PCR for detection of aminoglycoside resistance genes in enterococci. Antimicrob Agents Chemother. 2003:47(4):1423-6.

25. Choi JM, Woo GJ. Molecular characterization of high-level gentamicinresistant Enterococcus faecalis from chicken meat in Korea. Int J Food Microbiol. 2013;165(1):1-6.

26. Al-Talib H, Zuraina N, Kamarudin B, Yean CY. Genotypic variations of virulent genes in Enterococcus faecium and Enterococcus faecalis isolated from three hospitals in Malaysia. Adv Clin Exp Med. 2015;24(1):121-7.

27. Chanos $P$, Williams DR. Anti-listeria bacteriocin-producing bacteria from raw ewe's milk in northern Greece. J Appl Microbiol. 2011;110(3):757-68.

28. De Vuyst L, Foulquie Moreno MR, Revets H. Screening for enterocins and detection of hemolysin and vancomycin resistance in enterococci of different origins. Int J Food Microbiol. 2003;84(3):299-318.

29. Zeighami H, Valadkhani F, Shapouri R, Samadi E, Haghi F. Virulence characteristics of multidrug resistant biofilm forming Acinetobacter baumannii isolated from intensive care unit patients. BMC Infect Dis. 2019; 19:629.

30. Baldir G, Ozturk Engin D, Kucukercan M, Inan A, Akcay S, Ozyurek S, Aksaray S. High level resistance to aminoglycoside, vancomycin and linezolid in enterococci strains. J Microbiol Infect Dis. 2013;3(3):100-3.

31. Cekin Y, Erman Daloğlu A, Oğünç D, Ozhak Baysan B, Dağlar D, Inan D, Mutlu D, Ongüt G, Colak D. Evaluation of vancomycin resistance 3 multiplexed PCR assay for detection of vancomycin-resistant enterococci from rectal swabs. Ann Lab Med. 2013;33(5):326-30.

32. Naserpour Farivar T, Najafipour R, Johari P, Aslanimehr M, Peymani A, Jahani Hashemi H, Mirzaui B. Development and evaluation of a Quadruplex Taq man real-time PCR assay for simultaneous detection of clinical isolates of Enterococcus faecalis, Enterococcus faecium and their vanA and vanB genotypes. Iranian J Microbiol. 2014;6(5):335-40.

33. Zeighami H, Haghi F, Masumian N, Hemmati F, Samei A, Naderi G Distribution of Integrons and gene cassettes among Uropathogenic and Diarrheagenic Escherichia coli isolates in Iran. Microb Drug Resist. 2015;21(4): 435-40.

34. Kafil HS, Asgharzadeh M. Vancomycin-resistant Enteroccus faecium and Enterococcus faecalis isolated from education hospital of Iran. Maedica. 2014; 9(4):323-7.

35. Padmasini E, Padmaraj R, Ramesh SS. High level aminoglycoside resistance and distribution of aminoglycoside resistant genes among clinical isolates of Enterococcus species in Chennai, India. Sci World J. 2014;2014:329157.

\section{Publisher's Note}

Springer Nature remains neutral with regard to jurisdictional claims in published maps and institutional affiliations.

Ready to submit your research? Choose BMC and benefit from:
- fast, convenient online submission
- thorough peer review by experienced researchers in your field
- rapid publication on acceptance
- support for research data, including large and complex data types
- gold Open Access which fosters wider collaboration and increased citations
- maximum visibility for your research: over 100M website views per year
At BMC, research is always in progress.
Learn more biomedcentral.com/submissions

